\title{
Optimal Wiring on Rectangular Structure
}

\author{
Pawan Kumar Patel \\ Department of Computer \\ Science and Engineering \\ Indian Institute of Technology \\ Kanpur, India
}

\author{
Rohit Kumar \\ Department of Electrical \\ Engineering \\ Indian Institute of Technology \\ (BHU) Varanasi, India
}

\author{
Nikita Gulati \\ Department of Computer \\ Science and Engineering \\ Lucknow Model Institute of \\ Technology Lucknow, India
}

\begin{abstract}
In this paper we worked upon on optimal wiring on rectangular structure. Here we are given a rectangle partitioned into smaller rectangles by axis-parallel line segments. Find a subset of the segments such that the resulting structure from these segments is connected and it touches every smaller rectangle.

Here we reduce the problem of exact cover by 3-sets (X3C), which is known to be NP-complete, into this problem and thus claim wiring problem to be NP-hard. This problem carries a special importance because very few problems in the domain of geometry are known to be NP-hard.
\end{abstract}

\section{Keywords}

Wiring on rectangular structure, NP-hard, Computational geometry, Graph theory

\section{INTRODUCTION}

Given a rectangle partitioned into smaller rectangles by horizontal and vertical line segments, find a set of the linesegments which touches each rectangle at least at one point on its boundry and these segments are connected (i.e., there is a path between any two points of these segments ). The optimization criterion is to minimize the sum of lengths of these segments.

An obvious application of this problem is to how to wire a building using minimum wire. Same applies to laying cooling or heating channels. Another application is in connecting modules of a VLSI chips.

\section{NP HARDNESS Of WIRING PROBLEM}

\subsection{Wiring Problem}

A floorplan is a rectangle in a plane which is partitioned by horizontal and vertical line segments such that each region is also a rectangle. For convenience treat it as a graph where the vertex set is the collection of the corners of all the rectangles and edges are the line segments between the vertices in the floor plan. A side is a line segment which connects two corners of the same rectangle. In general a side may contain more than one edge.

The wiring problem is to compute a minimum length connected subgraph of a floorplan (i.e., total length of the edges of the subgraph be minimum) which contains at least one vertex on the boundry of every rectangle. Observe that it will always be a tree. See figure 1 . In this section we shall show that this problem is NP-hard by reducing 3-set-exactcover problem. The proof is adapted from the proof of hardness of steiner tree computation for geometric rectilinear graph by Garey, Graham, and Johnson[1].

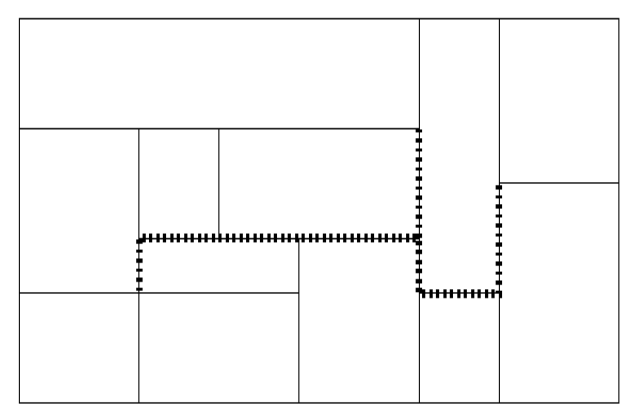

Figure 1: A floorplan and its solution

\subsection{3-set-Exact-Cover (X3C) Problem}

Given a family, $\mathrm{F}=\{\mathrm{F} 1, \mathrm{~F} 2, \ldots . . \mathrm{Ft}\}$, of 3 -element subsets of a universal set $U$ of $3 n$ elements, decide if there exists a subfamily F' C F of pairwise disjoint sets such that the union of all members of $F^{\prime}$ is equal to $U$. This problem is NP_complete[2]. We will prove the hardness of the wiring problem by transforming $\mathrm{X} 3 \mathrm{C}$ into it.

\subsection{The overall plan}

Let $\mathrm{F}=\{\mathrm{F} 1, \mathrm{~F} 2, \ldots . . \mathrm{Ft}\}$ be an input to the $\mathrm{X} 3 \mathrm{C}$ (3-set exact cover) problem, where the universal set is assumed to be the integer set $\{1,2, \ldots \ldots, 3 \mathrm{n}\}$, we will construct a floorplan Pi associated with set Fi for each i. Each plan will be of the same dimensions. Then we shall join them side by side along the $\mathrm{X}$ axis to form a single floor-plan $\mathrm{P}$ for the given $\mathrm{F}$.

We will show that there is a polynomial $L(n, t)$ such that the length of the wiring tree for $\mathrm{P}$ will be less than or equal to $\mathrm{L}(\mathrm{n} . \mathrm{t})$ if and only if the given $\mathrm{F}$ has an exact cover. It will also be shown that an exact cover can be extracted from such a solution tree in $\mathrm{O}(\mathrm{L}(\mathrm{n}, \mathrm{t}))$ time.

\subsection{Construction of $\mathbf{P i}$}

We first introduce two gadgets, junction and crossover, which are the bulding blocks of the floor-plans Pi. Figure 1 shows the gadgets. There are two variants of crossover, standard and warped. The length parameters used in describing the gadgets are $K=162 \mathrm{qnt}+2888 \mathrm{n} 2 \mathrm{t} \_9 \mathrm{n}+1$ and $\epsilon$ which will define later. It will be helpful to remember that $\epsilon<<1$.

Symbol $\mathrm{q}$ is defined to be sum of $(\mathrm{ai}+\mathrm{bi}+\mathrm{ci})$ for $\mathrm{i}=1$ to $\mathrm{t}$, where $\mathrm{Fi}=\{$ ai, bi, ci $\}$.

Each junction has one and each crossover has two active regions which are highlighted by shading in figure 1 .

The floorplan $\mathrm{Pi}$ associated with the set $\mathrm{Fi}=\{4,1,2\}$ is shown in figure 3 , where a junction at the bottom is connected to three stacks of crossover of heights ai, bi, ci respectively. 


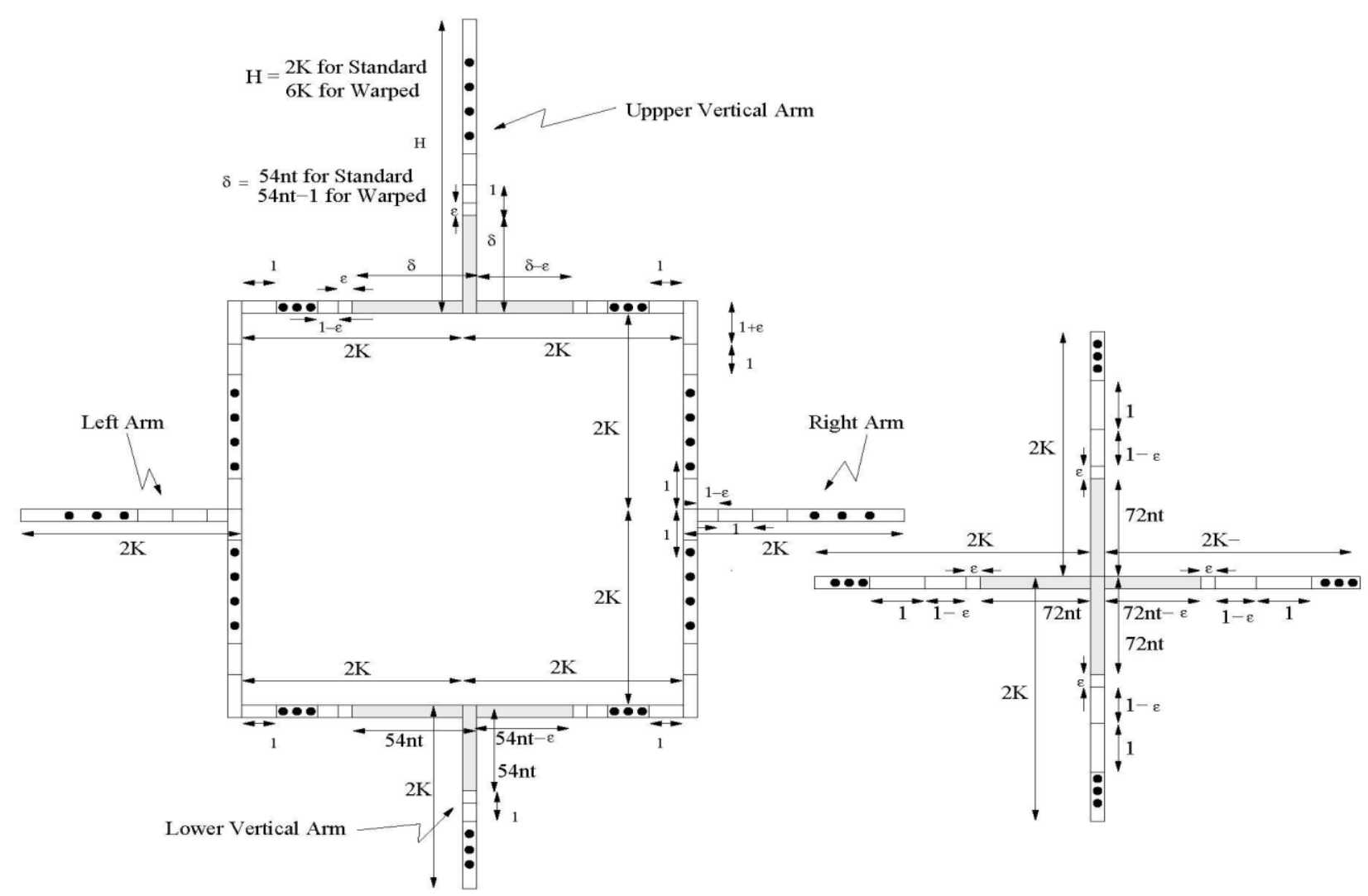

Figure 2: Crossover and Junction

The floorplan $\mathrm{P}$ associated with $\mathrm{F}$ is constructed by placing $\mathrm{P}_{1}, \mathrm{P}_{2}, \ldots \ldots \ldots$.Pt side by side so that right side of $\mathrm{P}_{\mathrm{i}}$ coincides with the left side of $\mathrm{P}_{i+1}$. In addition, a stack is attached to the left of the figure consisting of $8 \mathrm{~K}$ rectangles of size $1 \mathrm{x} \in$ and one $24 \mathrm{nK} x \in$ rectangle at the top of the stack, see figure 4 for $\mathrm{P}_{1}$ with the additional stack at the left wall. One way $\mathrm{P}_{1}$ and $\mathrm{P}_{\mathrm{t}}$ differ from other $P_{i}$ is that the leftmost rectangles in the horizontal chain of small rectangles in $P_{1}$ end with an $\epsilon x \in$ rectangle. This is the case for all but the bottom two chains. In case of $P_{t}$ the rightmost rectangles of all but the bottom chain is $\epsilon \mathrm{X} \epsilon$. Figure 5 shows complete $\mathrm{P}$. It uses $\mathrm{q}$ crossovers and $\mathrm{t}$ junctions.

\subsection{Optimal wiring tree for $P$}

In this section we will determine some properties of any optimal solution of the wiring on $\mathrm{P}$ which are crucial for the proof. In the following section we use these properties to show that the sum of the lengths of the edges in the optimal solution, will be less than or equal to L(n.t) if and only if the underlying $\mathrm{X} 3 \mathrm{C}$ problem has a solution.

\subsubsection{Coverage of the smaller rectangles}

Let us partition the rectangles of $P$ into 3 classes: $R_{0}$ which have longer side upto $1+\epsilon ; R_{1}$ which have longer sides in the range from $54 \mathrm{nt}-1$ to $72 \mathrm{nt}$; and $\mathrm{R}_{2}$ have each side at least $2 \mathrm{~K}$ $\epsilon$ in length. This partitions all rectangles of $P$ except the top rectangle at the left boundry. This $\epsilon \mathrm{x} 24 \mathrm{nK}$ rectangle is also included in class $R_{2}$. Observe that $R_{1}$ are precisely the rectangles in the active regions. We further partition $R_{0}$ into terminal and non terminal rectangles, where the former contains all the $\epsilon \mathrm{x} \epsilon$ sized rectangles. Observe that at least one vertex of each smaller side of a no-terminal rectangle is shared by other $\mathrm{R}_{0}$ rectangles. We shall denote these subclass by $R_{0 t}$ and $R_{0 n}$ respectively. Terminal rectangles are attached to the left side of $P_{1}$, right side of $P_{t}$, and to each $R_{1}$ rectangle.

In the similar fashion as for the rectangles partition the edges also into 3 classes: $\mathrm{E}_{0}$ consists of edges of length not exceeding $1+\epsilon ; \mathrm{E}_{1}$ are the edged with length between $54 \mathrm{nt}-1$ to $72 \mathrm{nt}$; and $\mathrm{E}_{2}$ have all the larger edges. Verify that each edge of $E_{2}$ is longer than $K$.

In order to establish polynomiality of this transformation from $\mathrm{X} 3 \mathrm{C}$, we need to determine some counts. Break the $\mathrm{R}_{0 \mathrm{n}}$ class into four subclasses, see figure 6 , and denote the number of (i) $1 \mathrm{x} \in$ rectangles with 4 vertices by $\mathrm{m}_{1}$, (ii) those with 5 vertices by $m_{2}$, (iii) $(1-\epsilon) \mathrm{x} \epsilon$ sized rectangles by $\mathrm{m}_{3}$, and (iv) $(1+\epsilon) \times \epsilon$ sized rectangles by $m_{4}$. The number of terminal rectangles will be denoted by $\mathrm{m}_{0}$. A trivial but cumbersome exercise gives $m_{0}=6 q+6 n+4 t+1, m_{1}=14 K q+42 K t+$ $72 \mathrm{Kn}+65 \mathrm{~K}-324 \mathrm{qnt}-288 \mathrm{nt}^{2}-10 \mathrm{t}+\mathrm{q}-\mathrm{n}-1, \mathrm{~m}_{2}=2 \mathrm{q}+5 \mathrm{t}+1$, $\mathrm{m}_{3}=6 \mathrm{n}+7 \mathrm{t}+8 \mathrm{q}+1$, and $\mathrm{m}_{4}=4 \mathrm{q}+\mathrm{t}+1$. Since $\mathrm{q}<9 \mathrm{nt}$, each of these numbers is a polynomial in $n$, $t$. Each $R_{0 n}$ rectangle has two major edges which are parts of the longer sides and which have length between $1-\epsilon$ and $1+\epsilon$.

In this paper we adopt a convention in which the same symbol will represent a set of edges as well as the graph induced by those edges, depending on the context.

Symbol T will denote an optimal wiring tree on $\mathrm{P}$, i.e. solution of the wiring problem on $\mathrm{P}$. The limiting case of the floorplan $P$, where $\epsilon=0$, will be denoted by $P^{\prime}$, i.e., $P^{\prime}=\lim _{\epsilon \rightarrow 0} P$. 
Henceforth $\mathrm{P}$ will denote the complete floorplan with $\epsilon=$ $1(20 q+23 n+28 t+9)$

$\mathrm{T}_{0}$ will denote $\mathrm{T} \cap \mathrm{E}_{0}$ and $\mathrm{T}_{1}=\mathrm{T}-\mathrm{T}_{0}$. Observe that the residue of each $\mathrm{R}_{0}$ rectangle in $\mathrm{P}^{\prime}$ is an edge of length 1 . By T' denote $\lim _{\epsilon \rightarrow 0} T$. Similarly by $T_{0}^{\prime}$ and $T_{1}^{\prime}$ denote the residue of $T_{0}$ and $\mathrm{T}_{1}$ in $\mathrm{P}^{\prime}$.

Observation 1 Every cycle in $\mathrm{P}$ has at least one edge of size greater than $\epsilon$.

Observation 2 Each cycle in $\mathrm{P}^{\prime}$ contains an edge which is the residue of the longer side of an $\mathrm{R}_{1}$ rectangle, i.e., an active region rectangle.

Observation 3 There are $4 q+8 t+9 n+2$ rectangles and each of these rectangles has at least one $\mathrm{R}_{0 \mathrm{n}}$ rectangle adjacent to it.

Proposition 4 Let $X$ be an $R_{0}$ rectangle and $C$ be a cycle in $P$. Let $\mathrm{C}$ contain a major edge e of $\mathrm{X}$ which is contained in side $s$. Let $s$ be the side parallel to $s$ in $X$. Then (i) $s$ is a longer side of $\mathrm{X}$, (ii) either $\mathrm{C}$ contain $\mathrm{s}$ ' or it contains a longer side of an $R_{1}$ rectangle.

Proof (i) Major edges are contained in the longer sides of a rectangle.

(ii) As we take the limit $\lim _{\epsilon \rightarrow 0}$, line segments e, $s$, and s' will coincide with a single segment, say s" in $\mathrm{P}$ '. Let $\mathrm{C}$ reduce to $\mathrm{C}^{\prime}$ in $\mathrm{P}$ '. Then the edges of $\mathrm{C}^{\prime}$ can be partitioned into simple cycles and simple paths. If $\mathrm{s}$ " is in a simple path, then s' must be on C. On the other hand, If s" is in a cycle in C', then due to the previous observation the longer side of an $\mathrm{R}_{1}$ rectangle must be on $\mathrm{C}$.

Lemma $5 \mathrm{~T}$ contains at least one major edge from each $R_{0 n}$ rectangle.

Proof Let $\mathrm{X}$ be a non terminal rectangle of $\mathrm{R}_{0}$ which does not satisfy the claim. By the definition,, $\mathrm{X}$ has two adjoining $\mathrm{R}_{0}$ rectangle which are separated by more than $\epsilon$. Label them by $\mathrm{Y}$ and $\mathrm{z}$. Since $\mathrm{T}$ touches all three rectangles, $\mathrm{Y}^{\prime}=\mathrm{T} \cap(\mathrm{Y} \mathrm{U}$ $X)$ and $Z^{\prime}=T \cap(Z U X)$ are non-empty where the $X, Y, Z$ may be treated as the sets of the edges on their sides. Since $T$ does not contain any major edge of $\mathrm{X}, \mathrm{Y}^{\prime}$ and $\mathrm{Z}^{\prime}$ must be unconnected. Let y belongs to $\mathrm{Y}^{\prime}$ and $\mathrm{z}$ belongs to $\mathrm{Z}^{\prime}$ be a pair of vertices which are closest to each other.

Add the shortest path between $\mathrm{y}$ and $\mathrm{z}$ in $\mathrm{P}$, to $\mathrm{T}$. Let the resulting subgraph be called $\mathrm{H}$. This added path must contain a major edge $\mathrm{e}$ of $\mathrm{X}$ and the length of the path canot exceed $3 x(1+\epsilon)+\epsilon$ since sides of each rectangle is at most $1+\epsilon$ and their width is $\epsilon$. The subgraph $\mathrm{H}$ has a cycle (since $\mathrm{T}$ is a tree) and the cycle contains e. From proposition 4 either T contains one major edge of $\mathrm{X}$ or one longer side of a $\mathrm{R}_{1}$ rectangle. The former is not possible from the assumption. Therefore $\mathrm{T}$ must contain a longer edge $p$ of an $R_{1}$ rectangle. By deleting $p$ from $\mathrm{H}$ we again get a tree, call it $\mathrm{H}^{\prime}$, and it also touches all the rectangles. Therefore this is also a candidate of wiring solution. Since the length of $\mathrm{p}$ is at least $54 \mathrm{nt}-1$ and the added edges are at most $3+4 \mathrm{c}$ in length, $\mathrm{H}^{\prime}$ has lesser length. This implies that $\mathrm{T}$ is not an optimal solution, which is contradiction.

In a wiring solution if a wire connects diagonally opposite vertices of a rectangle then it has two options of equal cost first horizontal then vertical side or its converse. This way we can delay a traversal along an $\epsilon$ edge on a non-terminal rectangle until a 5-vertex rectangle is reached. Call such wiring solution normalized, see figure 7 . Using this observation and lemma 5 we have following result.
Corollary 6 The cost of $\mathrm{T}_{0}$ is between $\mathrm{L}_{0}=\mathrm{m}_{1}+\mathrm{m}_{4}+(1-\epsilon)$ $\left(\mathrm{m}_{2}+\mathrm{m}_{3}\right)$ and $\mathrm{L}_{0}+\epsilon(28 \mathrm{q}+21 \mathrm{n}+28 \mathrm{t}+8)$.

Proof The smaller major edge of five vertex $1 \mathrm{x} \epsilon$ is $1-\epsilon$ long. The smaller major edge of a $(1+\epsilon) \mathrm{x} \epsilon$ rectangle has length 1 . Using these fact and the lemma we directly get the lower bound. For the upper bound first delete all the $\epsilon$ edges which are pendants ( having one vertex of degree 1 ) from $T_{0}$. As observed earlier, the reduced graph can be transformed into normalized from without any extra cost. So assume that the reduced $\mathrm{T}_{0}$ is normalized.

Then the cost due to five vertex rectangles can be upto $1+\epsilon$ for $1 \mathrm{x} \epsilon$ rectangle and $1+2 \epsilon$ for $(1+\epsilon) \mathrm{x} \epsilon$ rectangles. In addition the solution may cover terminal rectangles. So the cost can increase upto $2 \epsilon\left(\mathrm{m}_{0}+\mathrm{m}_{2}+\mathrm{m}_{4}\right)$. To this we add the cost of the pendants. The only purpose for the pendants will be to touch the $R_{2}$ rectangles as all others are already in contact of $T_{0}$. This can add at most $4 q+8 t+9 n+2 \epsilon$ edges to $\mathrm{T}_{0}$.

Observation 7 The graph induced by $\mathrm{R}_{0}$ rectangles has $2 \mathrm{q}+$ $3 n+1$ connected components.

This observation and lemma 5 imply that the subgraphs induced by $T_{0}$ must have at least $2 q+3 n+1$ components.

Lemma $8 T_{0}$ has exactly $2 q+3 n+1$.

Proof Assume that the number of components is greater than $2 q+3 n+1$. Then there must be at least two components of $T_{0}$ in the same component of $\mathrm{R}_{0}$.

Consider two such $\mathrm{T}_{0}$ components. If there is a $\mathrm{R}_{0 \mathrm{n}}$ rectangle in this component such that each of its major edge belongs to some $\mathrm{T}_{0}$ component. Then these $\mathrm{T}_{0}$ components are separated by $\epsilon$ distance. In case no $\mathrm{R}_{0 \mathrm{n}}$ rectangle contributes its major edge to more than one component, then there must be a rectangle whose one vertex is touched by one $T_{0}$ component and one major edge belongs to another, see figure 8 . Sice the distance between a vertex and a major edge is at most $2 \epsilon$ (in a 5 vertex rectangle), the two components are separated by a path of at most $2 \epsilon$ length. In other words, their closest points are separated by a path of at most $2 \epsilon$ length. Add this path to $\mathrm{T}$, which will create a cycle. Delete a non- $\epsilon$-edge from this cycle. The resulting tree connects the same set of vertices (or one more) as does $\mathrm{T}$ therefore it is also a candidate solution for the wiring problem. This tree costs lesser than T since $2 \epsilon$ $<1-\epsilon$. but this is not possible since $\mathrm{T}$ is optimal.

Corollary 9 There is one $\mathrm{T}_{0}$ component in each $\mathrm{R}_{0}$ component.

Lemma 10 T' has no cycles.

Proof We know that $\mathrm{T}$ is a tree. Assume that $\mathrm{T}$ ' has a cycle. Then $\mathrm{T}$ must have two vertices which are separated by a path $\mathrm{S}$ of $\epsilon$-edges which is not a part of T. The longest such path in $P$ has length $2 \epsilon$. Then T U S will have a cycle which contains S. Once again, as in the proof of Lemma 8 we can construct another solution of the wiring problem which costs less. Therefore the assumption must be wrong.

\subsection{Connecting the components of $\mathbf{T}_{0}$}

In this section we will establish the underlying $\mathrm{X} 3 \mathrm{C}$ problem has an exact cover if and only if $\mathrm{T}$ costs less than $\mathrm{L}=\mathrm{L}_{0}+$ $162 n t q+288 n^{2} t-9 n+1$. Also if there is a solution of the wiring problem in this range, then a solution of $\mathrm{X} 3 \mathrm{C}$ problem can be constructed in $\mathrm{O}(\mathrm{L})$ time. The immediate consequence of these claims is that if wiring problem has a polynomial 
time solution, then $\mathrm{X} 3 \mathrm{C}$ can also be solved in polynomial time since $\mathrm{L}$ is a polynomial in $\mathrm{n}, \mathrm{t}$.

In order to construct the solution tree we need to include the edges in addition to $\mathrm{T}_{0}$ so that the resulting graph becomes connected. Denote the set of these edges by $\mathrm{T}_{1}$. Irrespective of any other criterion/consideration these edges must provide connections between $2 q+3 n+1$ components of $T_{0}$ graph. The additional edges will be from $E_{1} U_{2}$. Each $E_{2}$ edge is at least $\mathrm{K}$ in length. We will show that this renders it too expensive to use. The $\mathrm{T}_{1}$ edges belonging to an active region become responsible to connect the $\mathrm{T}_{0}$ component surrounding the region. Therefore these edges from 1 or 2 connections, depending upon whether they connect 2 or 3 components. Figure 9 shows possible patterns of these $\mathrm{T}_{1}$ edges. Table 1

shows the average cost of forming one connection under these patterns.

Table 1: Patterns for active region, number of connections and their costs

\begin{tabular}{|lllll|}
\hline Gadget & Pat. & Con. & Range of cost & Range of cost/conn. \\
\hline Warped & $\beta_{1}$ & 2 & $162 n t-3+.5 \epsilon \pm .5 \epsilon$ & $81 n t-1.5+.25 \epsilon \pm .25 \epsilon$ \\
Crossover & $\beta_{2}$ & 1 & $108 n t-2-.5 \epsilon \pm 1.5 \epsilon$ & $108 n t-2-.5 \epsilon \pm 1.5 \epsilon$ \\
& $\beta_{3}$ & 1 & $108 n t-2+\epsilon \pm \epsilon$ & $108 n t-2+\epsilon \pm \epsilon$ \\
\hline Standard & $\alpha_{1}$ & 2 & $162 n t+.5 \epsilon \pm .5 \epsilon$ & $81 n t+.25 \epsilon \pm .25 \epsilon$ \\
Crossover & $\alpha_{2}$ & 1 & $108 n t-.5 \epsilon \pm 1.5 \epsilon$ & $108 n t-.5 \epsilon \pm 1.5 \epsilon$ \\
& $\alpha_{3}$ & 1 & $108 n t+\epsilon \pm \epsilon$ & $108 n t+\epsilon \pm \epsilon$ \\
\hline Junction & $\gamma_{1}$ & 3 & $288 n t+.5 \epsilon \pm .5 \epsilon$ & $96 n t+.166 \epsilon \pm .166 \epsilon$ \\
& $\gamma_{2}$ & 2 & $216 n t+.5 \epsilon \pm .5 \epsilon$ & $108 n t+.166 \epsilon \pm .166 \epsilon$ \\
& $\gamma_{3}$ & 2 & $288 n t-\epsilon \pm \epsilon$ & $144 n t-.5 \epsilon \pm .5 \epsilon$ \\
& $\gamma_{4}$ & 1 & $144 n t-.5 \epsilon \pm 1.5 \epsilon$ & $144 n t-.5 \epsilon \pm 1.5 \epsilon$ \\
& $\gamma_{5}$ & 1 & $144 n t$ to $144 n t+2 \epsilon$ & $144 n t$ to $144 n t+2 \epsilon$ \\
\hline
\end{tabular}

Lemma 11 If $T_{1} C E_{1}$ then (i) the number of $\alpha_{1}$ and $\beta_{1}$ patterns cannot be more than $\mathrm{q}$.

(ii) if the number of $\alpha_{1}$ and $\beta_{1}$ patterns is equal to $q$, then the number of $\beta_{1}$ patterns cannot be more than 3 .

Proof (i) If there are more than q patterns of $\alpha_{1}$ and $\beta_{1}$ type, then at least one crossover will have two $\alpha_{1}$ s or one $\alpha_{1}$, and one $\beta_{1}$ (in case of top of the stack crossovers). Then T' will have a cycle, contradicting Lemma 10.

(ii) If $q$ connection of the type $\alpha_{1}$ and $\beta_{1}$ are used, then each crossover will have one of these connections, because as shown in part (i) same crossover cannot have two of these connections. This will connect all the chains of the same level. If more than $3 n \beta_{1}$-connections are used, then some level will have two or more connections. This will provide more than one connection between the horizontal chain of that level and that of the level immediately above. Once again it implies that T' has a cycle.

Lemma 12 Let $T_{1} C E_{1}$. If in a wiring tree the cost of $T_{1}$ is less than $162 q n t+288 n^{2} t-9 n+1$, then the $T_{1}$ will have $3 n$ patterns of $\beta_{1}$ type, q-3n patterns of $\alpha_{1}$ type and $n$ patterns of $\gamma_{1}$ type.

Proof Suppose in the given solution u connection (between a pair of $\mathrm{T}_{0}$ components) are due to $\beta_{1}$ patterns, $\mathrm{v}$ connections are from $\alpha_{1}$ patterns $\mathrm{w}$ connection from $\gamma_{1}$ patterns and remaining $2 \mathrm{q}+3 \mathrm{n}-\mathrm{u}-\mathrm{v}-\mathrm{w}$ connections due to other patterns. Then from the table $1 \mathrm{~T}_{1}$ must cost off at least (81nt-1.5) $\mathrm{u}+$ $(81 n t) w+(108 n t)(2 q+3 n-u-v-w)$. We are given that this cost is less than $162 q n t+288 n^{2} t-9 n+1$. Simplifying the inequality we get $(2 q+3 n-u-v-w) 12 n t+(2 q-u-v) 15 n t+9 n$ $-1.5 \mathrm{u}-1<0$. Sice there can be at most $3 \mathrm{t}$ patterns of $\beta_{1}$ type, $\mathrm{u} \leq 6 \mathrm{t}$. Thus $15 \mathrm{nt}>12 \mathrm{nt}>1.5 \mathrm{u}+1$. Lemmas 8 and 11 shows that $2 q+3 n-u-v-w$ and $2 q-u-v$ are non negative. If either of these quantities is positive then the left hand side expression will become positive. This requires that $2 q+3 n-u-v-w=0$ and $2 \mathrm{q}-\mathrm{u}-\mathrm{v}=0$. Then inequality simplifies to $1.5(6 \mathrm{n}-\mathrm{u})-1<$ 0 . As $2 \mathrm{q}-\mathrm{u}-\mathrm{v}=0$, from Lemma 11 we also know that $6 n-\mathrm{u} \geq$ 0 . Again observe that if $6 n-u$ is positive then this inequality will be unsatisfiable. So we must have $6 n-u=0$. So $u=6 n$, $v=2 q-6 n$ and $w=3 n$.

Corollary 13 If $T_{1} C E_{1}$ and $T_{1}$ costs less than 162qnt +288 $n^{2} t-9 n+1$, then $X 3 C$ problem has an exact cover.

Proof We have seen that $T_{1}$ consists of $3 n$ patterns of $\beta_{1}$ type, q-3n patterns of $\alpha_{1}$ type, and $n$ patterns of $\gamma_{1}$ type. As we have seen in the proof of Lemma 12 that every crossover has one $\beta_{1}$ or $\alpha_{1}$ pattern. This ensures that the $T_{0}$ components on horizontal arms of the same level are connected. If $\beta_{1}$ patterns are at the same level, say i, then there are two connections between levels $i$ and $i+1$ (above $i$ ). This will lead to a cycle in T' which is not possible. So each $\beta_{1}$ must be at a different level. This ensures that each level is connected to the upper level through the upper arm of a warped crossover.

Now we will argue that in any stack of crossovers either all active regions contributing to $\mathrm{T}_{1}$ are upper ones or all are lower ones. Suppose crossover $\mathrm{C}_{2}$ is directly above crossover $\mathrm{C}_{1}$. First assume that upper active region of $\mathrm{C}_{2}$ and lower 
active region of $\mathrm{C}_{1}$ share edges with $\mathrm{T}_{1}$. This will render the $\mathrm{T}_{0}$ component $\mathrm{I}$ the vertical arm between the two disconnected. In case the lower active region of $\mathrm{C}_{2}$ and the upper active region of $\mathrm{C}_{1}$ contribute edges to $\mathrm{T}_{1}$, then this will provide a connection between the two levels. In addition, the $\beta_{1}$ patterns at the level of $\mathrm{C}_{1}$ crossover also provides a connection between the same levels. This implies that T' will have a cycle, which is not possible.

Next we will show that $\beta_{1}$ patterns can occur only on those stacks which are associated with the junction having $\gamma_{1}$ pattern. Suppose the top crossover of a stack has $\beta_{1}$ where the corresponding junction does not have $\gamma_{1}$. Then that junction does not contribute any edge to $T_{1}$ This implies that the vertical arm of the junction corresponding to the stack remains unconnected since the stack above it will have upper active region with $\alpha_{1}$ (or $\beta_{1}$ ) pattern.

Therefore all $\beta_{1}$ pattern must be associated with only the stacks of junctions with $\gamma_{1}$ patterns. Since each $\beta_{1}$ pattern is at a different level, the sets corresponding the junctions having $\gamma_{1}$ patterns from an exact cover.

Now we are equipped to prove the main result.

Theorem 14 The underlying $\mathrm{X} 3 \mathrm{C}$ problem has an exact cover iff the wiring tree of the corresponding floorplan has a solution costing less that $\mathrm{L}_{0}+162 \mathrm{qnt}+288 \mathrm{n}^{2} \mathrm{t}-9 \mathrm{n}+1$.

Proof (only if ) In Corollary 6 we have seen that a $T_{0}$ can be constructed which touches all the rectangles, has $2 q+3 n+1$ components and costs $\mathrm{L}_{0}+\epsilon(28 \mathrm{q}+21 \mathrm{n}+28 \mathrm{t}+8)$. We construct $\mathrm{T}_{1}$ as follows.

Let us denote the junction of $\mathrm{P}_{\mathrm{i}}$ by $\mathrm{J}_{\mathrm{i}}$. Let the exact cover be $\mathrm{F}^{\prime}$ $=\left\{\mathrm{F}_{\mathrm{a} 1}, \ldots \ldots \ldots \ldots, \mathrm{Fa}_{\mathrm{n}}\right\}$.

Then a wiring tree can be formed by constituting $T_{1}$ with : $\beta_{1}$ pattern in every top crossover of all the three stacks of $J_{a i}$ for all $\mathrm{F}_{\mathrm{ai}}$ for all $\mathrm{F}_{\mathrm{ai}}$ belongs to $\mathrm{F}^{\prime}, \alpha_{1}$ pattern in the upper active regions of all the standard crossovers of these stacks; $\alpha_{1}$ patterns in the lower active regions in all the other crossovers; and $\gamma_{1}$ pattern on every $\mathrm{J}_{\mathrm{ai}}$ for all $\mathrm{F}_{\mathrm{ai}}$ belongs to $\mathrm{F}$. Total cost of $T_{1}$ will be no more than $3 n(162 n t+\epsilon-3)+(q-3 n)(162 n t+$ $\epsilon)+n(288 n t+2 \epsilon)$. It simplifies to $162 q n t+288 n^{2} t-9 n+\epsilon$ $(q+2 n)$. The total cost of $T$ is at most $L_{0}+162 q n t+288 n^{2} t-9 n$ $+\epsilon(29 q+23 n+28 t+8)$ which is less than $L$.

(if) suppose the optimal wiring tree costs less than L. From corollary 6 we know that $\mathrm{T}_{0}$ costs at least $\mathrm{L}_{0}$ so $\mathrm{T}_{1}$ must cost less than $162 q n t+288 n^{2} t-9 n+1$. From corollary 13 we know that the $\mathrm{X} 3 \mathrm{C}$ problem must have an exact cover.

Corollary 15 If wiring problem is in class $\mathrm{P}$, then $\mathrm{X} 3 \mathrm{C}$ is also in class $p$.

Proof Since floorplan has a polynomial number of edges (in terms of $n$ and $t$ ), it is possible to verify in $\mathrm{O}(|\mathrm{T}|)$ time whether there are $3 \mathrm{n}$ patterns of $\beta_{1}$ type, $2 \mathrm{q}-3 \mathrm{n}$ patterns of $\alpha_{1}$ type, and $\mathrm{n}$ patterns of $\gamma_{1}$ type. Also identify which junctions have the $\gamma_{1}$ patterns in the process. As we consequence we can construct an exact cover.

Theorem 16 The wiring problem on rectangular floorplan is NP-hard. 


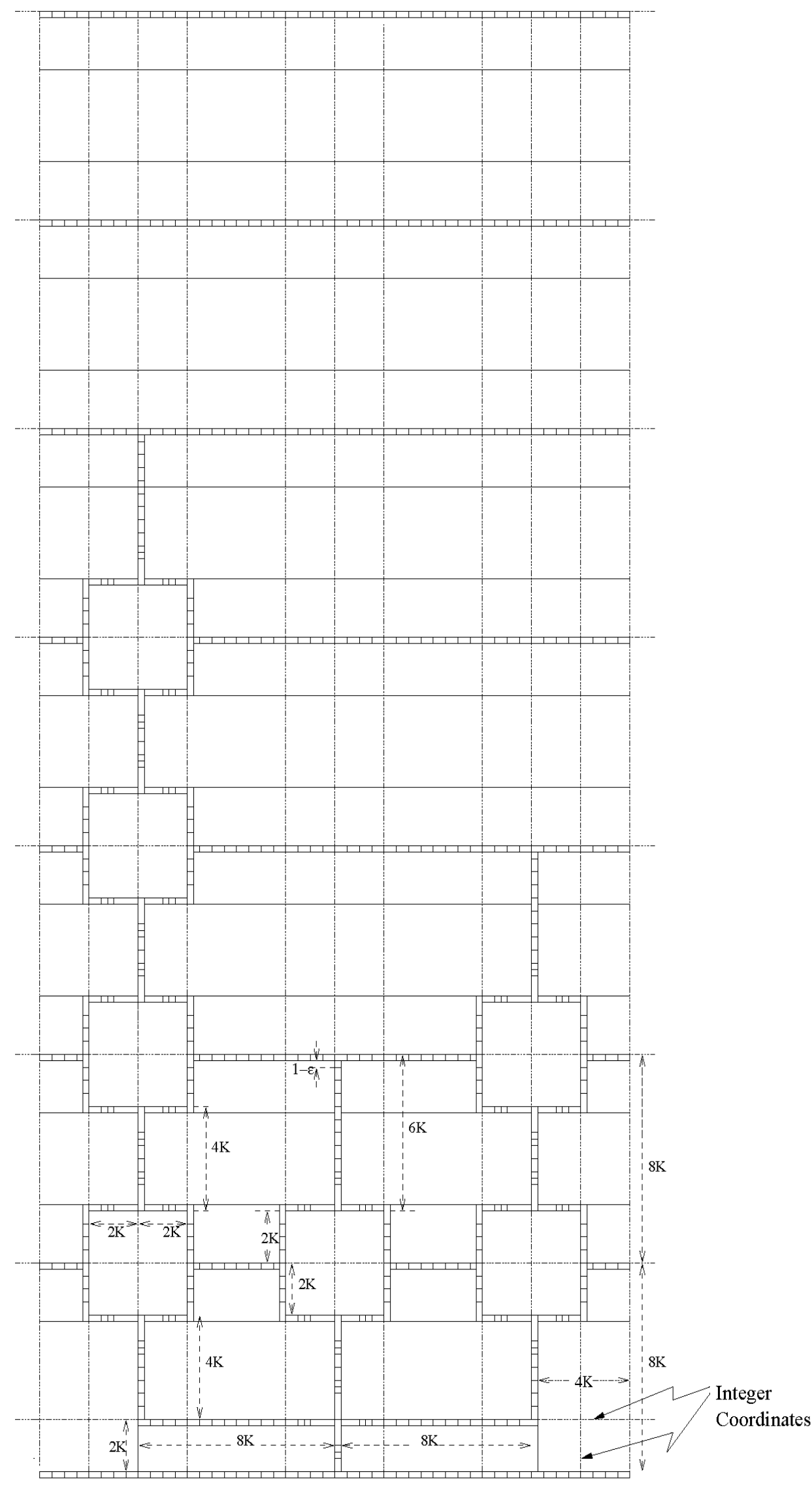

Figure 3: Floorplan of $P_{i}$ with $n=2$ and $F_{i}=\{4,1,2\}$ 


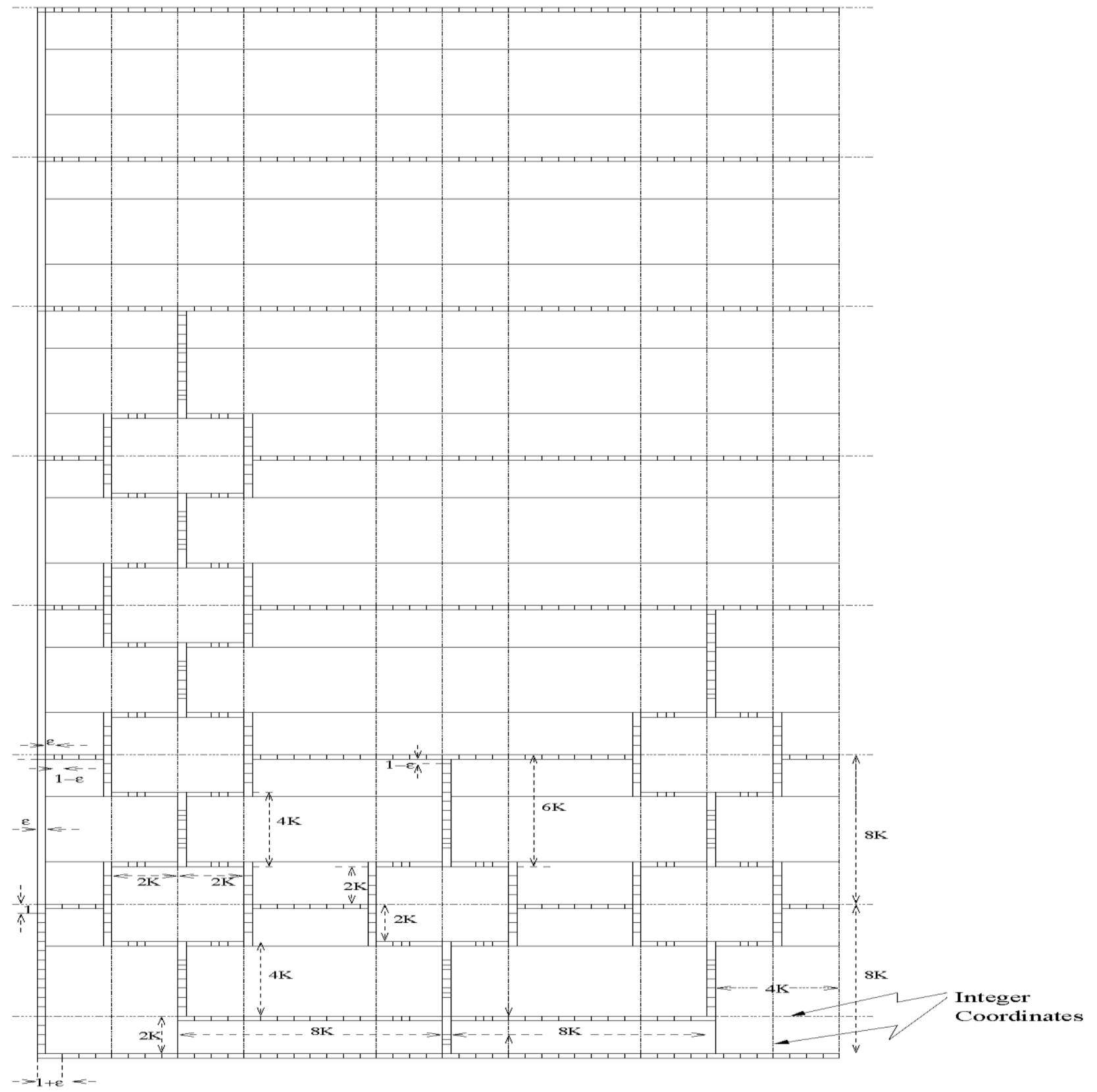

Figure 4: Floorplan of $p 1$ with stack of rectangles and terminals at the left

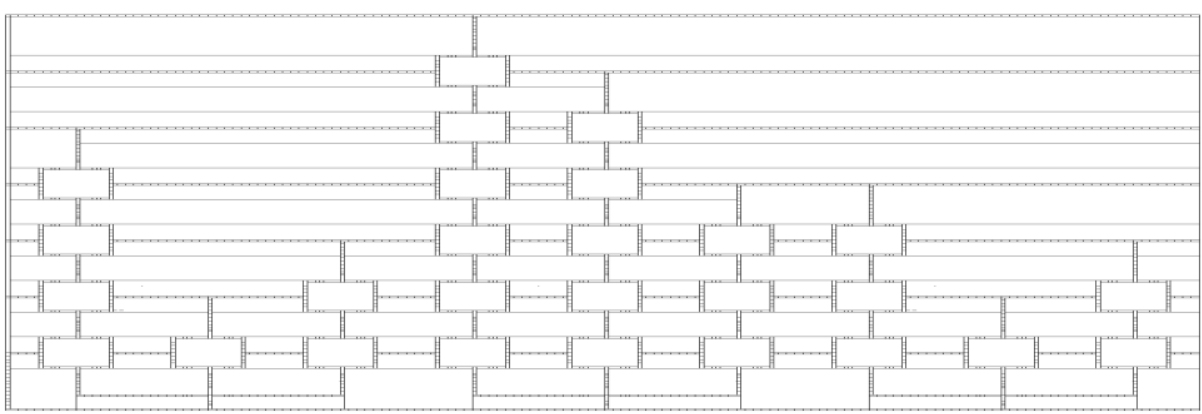

Figure 5: Floorplan of $P$ for $F=\{\{4,1,2\},\{6,5,3\},\{3,1,2\}\}$ 


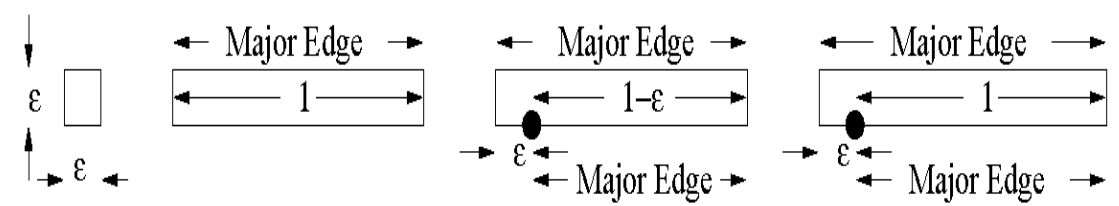

Figure 6: R0 Rectangles

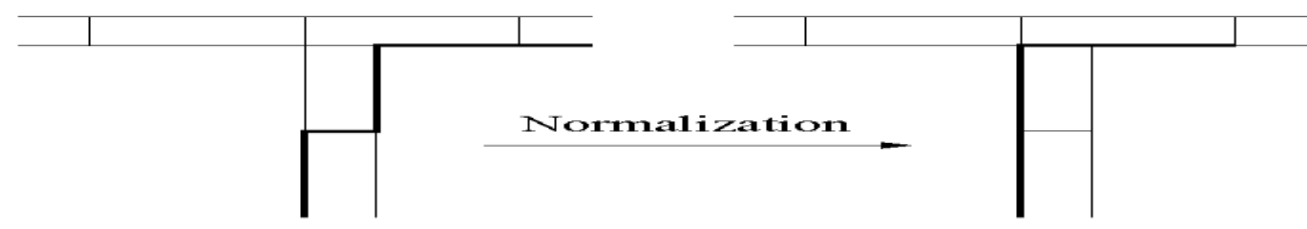

Figure 7: Tree Normalization
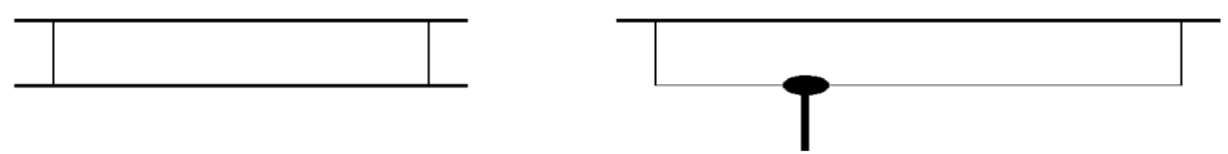

Figure 8: Two T0- components at the same R0 Rectangles

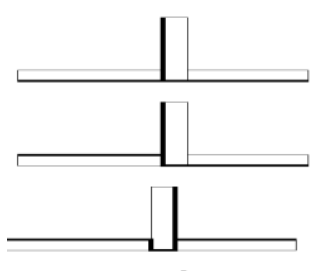

$\alpha 1 / \beta 1$

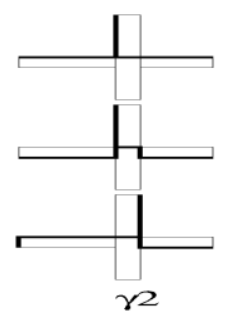

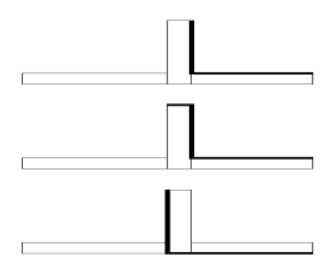

$\alpha 2 / \beta 2$

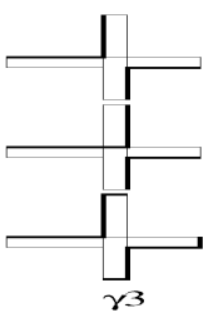

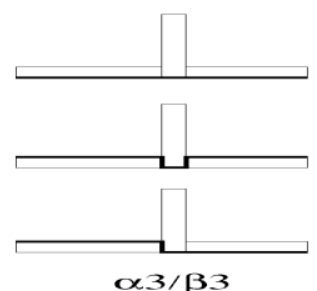

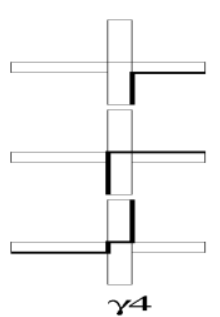

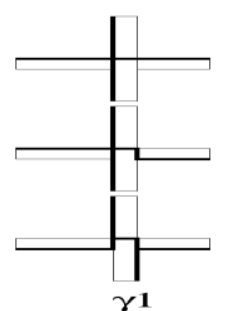

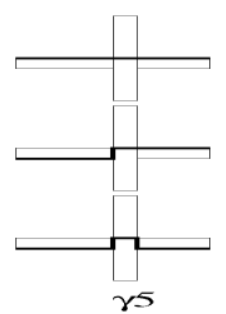

Figure 9 : Active region connection Patterns

\section{CONCLUSION}

In this paper we shows that wiring on rectangular floorplan is NP- hard problem by converting it into well known exact 3 cover (X3C). Due to wider application of this problem discuss above lead to think us, is there any good approximation algorithms that give good bound for this problem..

\section{REFERENCES}

[1]. M.R. Garey, R. L. Graham, and D.s. Johnson. Some npcomplete geometric problems. In STOC 76 : Proceeding of the eighth annual ACM symposium on theory of computing, page 10-22, New York, NY, USA, 1976. ACM Press.
[2]. R.M Karp. Reducibility among combinatorial problems. R. E. Miller and J. W. Thatcher, Plenum press, New York, USA, 1972.

[3]. S. Aaronson. Is P versus NP formally independent? Bulletin of the European Association for Theoretical Computer Science, 81, Oct. 2003

[4]. R. Impagliazzo, R. Paturi, and F. Zane, Which Problems Have Strongly Exponential Complexity?, Journal of Computer and System Sciences 63-4, (2001), pp. 512530 